\title{
Major depressive disorder during pregnancy: Psychiatric medications have minimal effects on the fetus and infant yet development is compromised
}

\author{
HANNA C. GUSTAFSSON,${ }^{a}$ SHERRYL H. GOODMAN,${ }^{b}$ TIANSHU FENG,${ }^{c}$ JEAN CHOI, ${ }^{c}$ SEONJOO LEE,${ }^{c, d}$ \\ D. JEFFREY NEWPORT, ${ }^{e}$ BETTINA KNIGHT, ${ }^{f}$ BLAIRE PINGETON,${ }^{d}$ ZACHARY N. STOWE, ${ }^{g}$ AND \\ CATHERINE MONK ${ }^{c, d}$ \\ ${ }^{a}$ Oregon Health and Science University; ${ }^{b}$ Emory University; ${ }^{c}$ New York State Psychiatric Institute; ${ }^{d}$ Columbia University Medical \\ Center; ${ }^{e}$ University of Miami Miller School of Medicine, ${ }^{f}$ University of Arkansas for Medical Sciences; and ${ }^{g}$ University of Wisconsin \\ at Madison, School of Medicine and Public Health
}

\begin{abstract}
Psychotropic medication use and psychiatric symptoms during pregnancy each are associated with adverse neurodevelopmental outcomes in offspring. Commonly, studies considering medication effects do not adequately assess symptoms, nor evaluate children when the effects are believed to occur, the fetal period. This study examined maternal serotonin reuptake inhibitor and polypharmacy use in relation to serial assessments of five indices of fetal neurobehavior and Bayley Scales of Infant Development at 12 months in $N=161$ socioeconomically advantaged, non-Hispanic White women with a shared risk phenotype, diagnosed major depressive disorder. On average fetuses showed the expected development over gestation. In contrast, infant average Bayley psychomotor and mental development scores were low $(M=84.10$ and $M=89.92$, range of normal limits 85-114) with rates of delay more than 2-3 times what would be expected based on this measure's normative data. Controlling for prenatal and postnatal depressive symptoms, prenatal medication effects on neurobehavioral development were largely undetected in the fetus and infant. Mental health care directed primarily at symptoms may not address the additional psychosocial needs of women parenting infants. Speculatively, prenatal serotonin reuptake inhibitor exposure may act as a plasticity rather than risk factor, potentially enhancing receptivity to a nonoptimal postnatal environment in some mother-infant dyads.
\end{abstract}

Increased scrutiny of antidepressant use during pregnancy has demonstrated several potential adverse outcomes for offspring based on large, epidemiological studies including elevated risk for attention-deficit/hyperactivity disorder (Clements et al., 2015), depression (Malm et al., 2016), and autism (Boukhris, Sheehy, Mottron, \& Berard, 2016). Psychiatric symptoms during pregnancy also are associated with compromised neurobehavioral development (Grote et al., 2010; O'Connor, Monk, \& Burke, 2016). However, studies commonly consider the effects of psychiatric medication use or depression that is untreated, with few studies including description of both possible exposures. Studies also

The authors gratefully acknowledge the women who participated in this study and the community obstetrical practices in the Atlanta area for their assistance. This study was supported by the Translational Research Center in Behavioral Sciences (TRCBS) Grant P50 MH077928. Research reported in this publication was also supported by the National Center for Advancing Translational Sciences of the National Institutes of Health under award number TL1TR002371. The content is solely the responsibility of the authors and does not necessarily represent the official views of the National Institutes of Health.

Address correspondence and reprint requests to: Catherine Monk, Behavioral Medicine/CUMC, 622 West 168th St. PH1540, New York, NY 10032; E-mail: cem31@columbia.edu. frequently compare children of prenatally medicated women who carry a psychiatric diagnosis to healthy controls, yet this is not an ecologically valid counterfactual. Findings from these studies sometimes do not replicate when the groups examined are medicated and unmedicated depressed women (e.g., Sujan et al., 2017), the more appropriate, yet rarely utilized, comparison. To address these concerns, the current study examined women who were homogenous with respect to a shared depression diagnosis, and we provide detailed information on treatment and symptoms.

Prenatal medication studies have largely focused on serotonin reuptake inhibitors (SRI), with limited assessment of other psychotropic medications, in particular, polypharmacy, though this approach too is less ecologically valid. While $10 \%$ of pregnant women are prescribed psychotropic medication, with antidepressant medications the most common, $1.6 \%$ use drugs from two or more different categories, typically combining an antidepressant and an anxiolytic (Ayad \& Costantine, 2015). Recently Bobo et al. (2012) reported that the majority of prenatal antiepileptic drug exposures are for nonepileptic conditions such as psychiatric symptoms. For the other major classes of psychotropic medications, there is data suggesting potential adverse effects on the developing child: bupropion increases uterine artery resistance, potentially 
compromising nutrient and oxygen transport (Monk et al., 2012); atypical antipsychotics and lamotrigine cross the placenta (Bank, Stowe, Newport, Ritchie, \& Pennell, 2017; Newport et al., 2007), and there is evidence of associations with delayed neurobehavioral development (Cummings, Stewart, Stevenson, Morrow, \& Nelson, 2011; Johnson, LaPrairie, Brennan, Stowe, \& Newport, 2012; Peng et al., 2013). In an important recent study, Salisbury et al. (2016) showed concomitant benzodiazepine and SRI use exacerbates poor neonatal adaptation.

The majority of studies on the potential impact of maternal psychotropic medication use on child outcomes do not evaluate children during the developmental stage when the effects are believed to occur, the fetal period. Yet the assessment of fetal behavior can provide unique insights as it disaggregates prenatal influences from postnatal confounds.

Expected neurobehavioral trajectories of fetal development have been established, and related to children's later functioning. As the fetal nervous system develops, fetal heart rate (FHR) and fetal movement (FM) both decrease; FHR variability (FHRV; an index of autonomic nervous system functioning) and the cross correlation of FHR and FM ("coupling," an indicator of neuroregulation) typically increase across gestation (DiPietro et al., 2004; Dipietro, Irizarry, Hawkins, Costigan, \& Pressman, 2001). Maturation of cardiac parasympathetic innervation accounts for the changes in FHR and FHRV (Dalton, Dawes, \& Patrick, 1983; Freeman, Garite, Nageotte, \& Miller, 2012), while development of the central nervous system and its coordination of the autonomic and somatic systems underlie the increase in coupling (Baser, Johnson, \& Paine, 1992; DiPietro, Hodgson, Costigan, \& Johnson, 1996; Dipietro et al., 2001, 2010). Differences in levels and developmental trajectories of these fetal metrics predict future development. For example, greater FHRV is associated with superior competence in mental, motor, and language development several years later (DiPietro, Bornstein, Hahn, Costigan, \& Achy-Brou, 2007). Similarly, greater FM has been related to less behavioral inhibition during the second year of life and, in male children, to higher activity levels (DiPietro et al., 2002; Doussard-Roosevelt, McClenny, \& Porges, 2001). Finally, in our work (Monk et al., 2004, 2011), greater third trimester FHR reactivity to in utero stimuli (operationalized as women's physiological reactivity during a laboratory stressor) is inversely associated with newborn resting-state brain connectivity between the amygdala and the dorsolateral prefrontal cortex (Posner et al., 2016), considered the mature pattern, and positively associated with more motorically reactive 4-month-old infant temperament (Monk et al., 2011; Werner et al., 2007).

In the few studies examining psychotropic medication use in relation to fetal development, SRI monotherapy $(n=96)$ versus unmedicated ( $n=37$ who discontinued SRI medication) compared to healthy controls without mental disorders $(n=130)$ showed greater overall FM at 15-19 and 27-29 gestational weeks but not at 37-39 weeks in the SRI versus other groups. Near term, fetuses in the medicated group compared to others had disrupted REM sleep (Mulder, Ververs, de Heus, \& Visser, 2011). In fetuses of SRI-only using women $(n=29)$ versus no psychotropic medication $(n=45)$, exposed fetuses showed reduced FHRV and middle cerebral artery pulsatility index (possibly suggesting compensatory maintenance of oxygen transport to vital organs such as the brain) at 36 gestational weeks (Rurak et al., 2011). In this report, unmeasured mental illness cannot be ruled out as a significant factor given the comparison groups. Neither of these studies assessed neurobehavioral development after birth.

Several reports show significant associations of psychotropic medications with decrements in early infant psychomotor, though not mental, development using the Bayley Scales of Infant Development (BSID) at ages 10-16 months old (Casper et al., 2011; Hanley, Brain, \& Oberlander, 2013). However, most of those studies relied on small sample sizes, that is, prenatal exposure groups ranging from 31 to 55 (Casper et al., 2011; Hanley et al., 2013). In a longitudinal study of 166 mother-infant dyads (SRI monotherapy $n=41$ ), untreated major depressive disorder (MDD; $n=27$, no exposure $n=98$ ), Santucci et al. (2017) showed SRI versus MDD and no exposure was associated with lower BSID psychomotor development at 6.5 and 13 months old; however, these differences were no longer observed at 19.5 months, and the lower scores were within the normal range. There were no findings for the mental development outcomes.

This study aimed to examine associations of maternal SRI and polypharmacy use with offspring neurobehavioral development both at the time of exposure (in utero as a fetus at 24, 30, and 36 weeks) and again at 12 months old in a sample of women with a shared risk phenotype, diagnosed depression, while also controlling for current mood symptoms, including anxiety given the tendency for prenatal and postnatal depression to co-occur with anxiety symptoms (Putnam et al., 2017). We reasoned that a medication effect identified in the fetal and 12-month-old assessments that showed fetal behavior as a mediator between prenatal medication exposure and infant development would indicate the influence of psychotropic medication on neurobehavioral development.

\section{Method}

\section{Overview}

The study was conducted at the Women's Mental Health Program at the Emory University School of Medicine. Pregnant women with lifetime histories of mental illness participating in a longitudinal investigation of the impact of perinatal maternal mood and stress on child neurobehavioral outcomes were screened for inclusion in the current analysis. The data reported here are part of a larger data set. Participants were enrolled prior to 16 weeks gestation, and evaluated at 4- to 6-week intervals. During their third trimester, they were invited to participate in a follow-up study. This report covers three pregnancy assessments, at gestational weeks $24 \pm 2,30 \pm 2$, and $36 \pm 2$ (first, second, and third testing sessions, respectively), 
four postpartum mood assessments, and one infant assessment session at 12 months. All symptom rating scales and medication use were coded with a HIPAA compliant identifier and entered into a centralized database. The Emory University Institutional Review Board approved the study, and all women provided informed consent.

\section{Participants}

Pregnant women ages 18-45 were referred by obstetrical and mental health care providers to a tertiary care program providing treatment to pregnant women with mental illness. At enrollment prior to 16 weeks gestation, current diagnoses were established using the Structured Clinical Interview for DSMIV Axis 1 Disorders (SCID; First, Spitzer, Gibbon, \& Williams, 1997). Following standard procedures, the SCID was used to determine the individual's principal current diagnosis as well as their lifetime history of additional Axis 1 disorders. Women were excluded from participation if they were actively suicidal, homicidal, exhibiting psychotic symptoms, carried a primary SCID diagnosis of psychotic disorder, had an active eating or substance use disorder within 6 months prior to conception, or had a medically complicated pregnancy. Of the 275 women enrolled who had fetal data, 161 were selected for inclusion in the current analyses based on a principal SCID diagnosis of MDD; women with a current primary SCID diagnosis of bipolar disorder were excluded from the current analysis. Forty-one (25.47\%) of the women in our analytic sample met SCID criteria for a lifetime history of generalized anxiety disorder. There were no differences in demographics (see Table 1) between the included and excluded participants. Of those included in the pregnancy sessions, $n=80$ agreed to participate in the postnatal followup study. There were no differences in those who elected follow-up versus not based on the same demographic variables as well as in prenatal mood symptoms and medication use during pregnancy.

\section{Medication use}

At each pregnancy testing session, a study physician (Z.N.S. or D.J.N.) completed a detailed weekly tracking sheet on the women's use of psychotropic medications. Women's dosage, timing, and number of medications was adjusted based on clinical symptoms and tolerability. Medications taken were: SRIs, bupropion, lamotrigine, atypical antipsychotics, benzodiazepines, and cardiovascular medications. Considering medication use as a non-mutually exclusive variable and as ever used during pregnancy, and listed here in order of frequency, 89 (55\%) women took an SRI, 22 (14\%) bupropion, $19(12 \%)$ a benzodiazepine, $9(6 \%)$ lamotrigine, $6(4 \%)$ an atypical antipsychotic, $13(8 \%)$ cardiovascular medication (i.e., anti-hypertensives and anti-thrombotics), and finally, $48(30 \%)$ did not take any psychiatric medication at any point in pregnancy. For each of the three pregnancy testing sessions, women were grouped into one of the following four categories: No Medication; SRI Only; SRI + Non-SRI; or Non-SRI. Medication group membership was considered as a time-varying effect in most analyses. See Figure 1 for additional information about the proportion of women in each of these medication use groups, including how group membership changed over time in a minority of individuals.

\section{Psychiatric symptoms}

Approximately every 4-6 weeks during pregnancy (including at the fetal testing sessions at 24,30, and 36 weeks gestation), a research interviewer masked to treatment status administered the 17-item Structured Interview Guide (Williams, 1988) for the Hamilton Rating Scale for Depression (HRSD-17; Hamilton, 1960) and the Hamilton Rating Scale for Anxiety (HRSA; Hamilton, 1959). Quarterly interrater reliability assessments were conducted throughout the study to maintain $\kappa$ statistics $>0.80$. These data were considered in two ways during analyses. Our primary models included the HRSD-17 and HRSA assessments that occurred concurrent to the fetal sessions (at 24, 30, and 36 weeks gestation). Because maternal depression and anxiety scores did not change significantly across the 24-, 30-, and 36-month assessments ( $p s=.70$ and .13 , respectively), we averaged symptom scores across these three visits to create our primary analysis variables. We considered measures of both depression and anxiety because, as noted above, a substantial number of women with depression during the perinatal period also report significant anxiety (Putnam et al., 2017).

In addition to these average scores, we also calculated variables that capture the fetuses' cumulative exposure to maternal symptomology: area under the curve (AUC) values were calculated using each of the serial symptom ratings using the trapezoidal method (standardized to a 40-week pregnancy). Separate AUC scores were calculated for HRSD-17 and HRSA. These AUC variables were used in the models examining fetal reactivity to a stressor (in addition to the average HRSD-17 and HRSA variables) based on previous studies that suggest that differences in FHR reactivity may vary as a function of cumulative exposure to maternal symptomatology (e.g., Monk et al., 2004). For postnatal mood, four assessments of mood symptoms were acquired and used to calculate HRSD-17 and HRSA AUC values that captured symptoms occurring across the first 12 months postpartum. Several previous studies have successfully used AUC values calculated using this method to capture cumulative exposure to maternal symptomatology over time (e.g., Lusby, Goodman, Bell, \& Newport, 2014; Monk et al., 2012).

\section{Urine drug screen}

For $141(88 \%)$ participants, we obtained urine drug screens during one fetal testing session (12\% of participants were randomly chosen for two screens) and tested for the following substances: amphetamines, barbiturates, cannabis, cocaine, and opiates. 
Table 1. Demographic and clinical characteristics of study sample by medication group

\begin{tabular}{|c|c|c|c|c|}
\hline \multirow{2}{*}{ Variable } & \multicolumn{4}{|c|}{ Mean $(S D)$ or $n(\%)$} \\
\hline & $\begin{array}{l}\text { No Medication } \\
\quad(n=43)\end{array}$ & $\begin{array}{l}\text { SRI Only } \\
(n=48)\end{array}$ & $\begin{array}{l}\text { SRI + Other } \\
\quad(n=27)\end{array}$ & $\begin{array}{l}\text { Other Only } \\
\quad(n=21)\end{array}$ \\
\hline Maternal age & $32.95(4.84)$ & $33.06(4.21)$ & $33.53(5.25)$ & $32.70(4.82)$ \\
\hline \multicolumn{5}{|l|}{ Race } \\
\hline White non-Hispanic & $37(86.05 \%)$ & $39(81.25 \%)$ & $27(100 \%)$ & $19(90.48 \%)$ \\
\hline African American & $5(11.63 \%)$ & $8(16.67 \%)$ & $0(0.0 \%)$ & $1(4.76 \%)$ \\
\hline Asian & $1(2.33 \%)$ & $1(2.08 \%)$ & $0(0.0 \%)$ & $0(0.0 \%)$ \\
\hline White Hispanic & $0(0.0 \%)$ & $0(0.0 \%)$ & $0(0.0 \%)$ & $1(4.76 \%)$ \\
\hline Native American & $0(0.0 \%)$ & $0(0.0 \%)$ & $0(0.0 \%)$ & $0(0.0 \%)$ \\
\hline Maternal education (years) & $16.35(1.97)$ & $16.38(2.01)$ & $16.19(1.90)$ & $16.33(1.96)$ \\
\hline Currently married & $38(88.37 \%)$ & $42(89.36 \%)$ & $23(85.19 \%)$ & $18(85.71 \%)$ \\
\hline Hollingshead $^{\mathrm{a}}$ & $50.00(7.73)$ & $49.81(9.49)$ & $50.56(7.87)$ & $51.05(9.17)$ \\
\hline Gravidity & $2.30(1.34)$ & $2.21(1.17)$ & $1.67(0.68)$ & $2.48(1.63)$ \\
\hline Parity & $0.84(0.72)$ & $0.60(0.68)$ & $0.37(0.49)$ & $0.95(1.20)$ \\
\hline Gestational age at birth & $38.68(1.30)$ & $38.04(2.26)$ & $38.21(2.31)$ & $37.88(1.80)$ \\
\hline Infant birthweight $(\mathrm{g})^{\mathrm{b}}$ & $3480(500)$ & $3070(535)$ & $3230(620)$ & $3090(390)$ \\
\hline Infant sex (\% female) & $13(38.24 \%)$ & $22(52.38 \%)$ & $12(44.44 \%)$ & $10(52.63 \%)$ \\
\hline Gestational diabetes & $2(5.71 \%)$ & $5(11.36 \%)$ & $3(11.11 \%)$ & $2(10.0 \%)$ \\
\hline Hydramnios & $1(2.86 \%)$ & $2(4.55 \%)$ & $2(7.41 \%)$ & $4(20.0 \%)$ \\
\hline Hyperesmesis & $2(5.71 \%)$ & $3(6.82 \%)$ & $2(7.41 \%)$ & $0(0.0 \%)$ \\
\hline Smoking & $6(17.14 \%)$ & $4(9.09 \%)$ & $6(22.22 \%)$ & $5(20.0 \%)$ \\
\hline
\end{tabular}

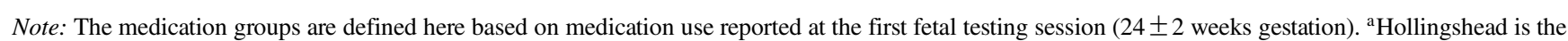
Hollingshead Four-Factor Index of Socioeconomic Status. ${ }^{b}$ Birthweight was significantly different among groups $(p=.006)$.

\section{Other health variables}

Information on participants' age, parity, gravidity, race/ethnicity, smoking, education, income, and marital status was obtained at study enrollment. Body mass index (BMI) was assessed via height at enrollment and weight at each fetal testing session. Structured abstraction of medical records was coded for pregnancy complications (gestational diabetes, hydramnios, hyperemesis, and smoking) as follows: none, at least one, or two (the maximum number).

\section{Fetal assessment}

Women were in a semirecumbent position as FM and FHR were acquired. As described in prior work (Doyle et al., 2015; Werner et al., 2007), to characterize indices of fetal development over gestation, women underwent the following protocol at each fetal testing session: resting baseline, a psychological stressor (a Stroop color-word task), and a resting recovery. Each of these task epochs lasted for $5 \mathrm{~min}$.

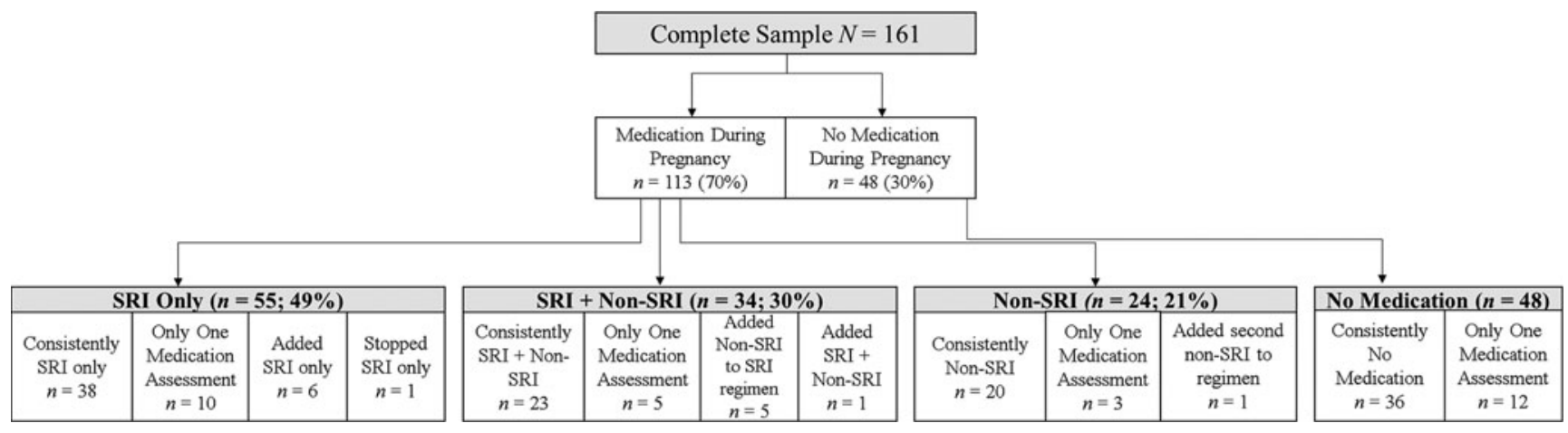

Figure 1. Maternal medication use during pregnancy. These classifications were based on maternal medication use at all three assessments (at $24 \pm 2,30 \pm 2$, and $36 \pm 2$ weeks gestation). The majority of women $(n=117 ; 73 \%)$ did not change their medication status over pregnancy, described above as "consistently" within that category. Thirty women (19\%) had only one medication assessment; their medication group membership was determined based on the medication information available. A minority of women $(n=14 ; 9 \%)$ changed medication groups over time (e.g., 6 women began the study not taking medication but added an SRI only). These women were categorized as delineated above. Maternal medication use was treated as time varying in the models (so group membership changes were accounted for in the models); this figure is meant to give a general sense of the types of medication use (and combinations of medication) reported in this sample over the course of the study. 
Data were obtained using a Toitu MT 325 fetal actocardiograph (Toitu Co., Ltd, Tokyo, Japan), which detects FM and FHR via a single transabdominal Doppler transducer. The fetal data were collected from the Toitu's output port, digitized at $50 \mathrm{~Hz}$ using a 16-bit $\mathrm{A} / \mathrm{D}$ card (National Instruments 16XE50) and analyzed offline without knowledge of medication or mood symptom status; the coupling of FM and FHR was calculated as previously described (Doyle et al., 2015; Monk et al., 2016). FHRV was calculated using the standard deviation of FHR (see Doyle et al., 2015). To verify effectiveness of the Stroop stressor paradigm, participants were asked to rate the stress they experienced on a 1 (none at all) to 10 (extreme stress) scale after each of the three periods (baseline, Stroop, and recovery).

\section{BSID—Second Edition (BSID-II) at 12 months old}

At infant age 12 months $(M=56.3$ weeks, range $=52-64$ weeks), doctoral students in clinical psychology or a research specialist administered the BSID-II following standard procedures. Administrators had completed several practice administrations with repeated feedback prior to engaging in study administration of the BSID. The infant was seated on the mother's lap, with the mother having been instructed to be as uninvolved as possible, unless asked otherwise by the test administrator. The developmental age for the Mental Developmental Index (MDI) and Psychomotor Developmental Index (PDI) were calculated following procedures in the BSID-II manual, including corrections for prematurity. Standardized scores have a mean of 100 and a $S D$ of 15 . Scores of 85-114 are considered within normal limits. Standardized, age-corrected scores were used in all analyses.

\section{Analysis}

To first characterize average fetal development across gestation, four unconditional hierarchical linear models (HLMs) were estimated, one for each fetal outcome variable (FHR, FHRV, FM, and coupling). Each model included random intercept and random slope terms, allowing for individual differences in both baseline levels and rates of change over time. To derive average Bayley scores, descriptive statistics were used.

To examine the effect of maternal prenatal psychotropic medication group on the four fetal variables over pregnancy, eight HLMs were estimated (four controlling for depression and an additional four for anxiety symptoms) based on fetal data collected from the 5-min baseline period. Each of these models included both the main effect of medication group (1-4 as described above) and their interaction with a term that captured the time-varying effect of medication group. Maternal symptomatology, assessed using the average HRSD-17 or HRSA values, was also included in these models. Because of concerns of multicollinearity (based on large, significant correlations between the various assessments of maternal depression and anxiety, $r \mathrm{~s}=.76-.82, p \mathrm{~s}<.0001)$, we considered average HRSD-17 and average HRSA scores in separate models. We examined pairwise group differences in fetal outcomes evaluated at the 24-, 30-, and 36-week testing sessions based on the estimated HLMs.

Fetal reactivity during the three different testing sessions also was examined using HLM with the three task periods (baseline, Stroop, and recovery) as a main effect and maternal stress rating as a time-varying covariate. We tested for an effect of medication group on changes in FHR during maternal engagement with the Stroop stressor task by adding interaction terms that captured the interaction between medication group and task period. Maternal mood rated by either the average HRSD-17 or the average HRSA was included in the models. In supplemental analyses, we elected to integrate measures of cumulative symptom exposure (i.e., HRSD-17 and HRSA AUC values) into these FHR reactivity models based on previous studies that suggest that differences in fetal heart rate reactivity may vary as a function of cumulative, rather than acute, exposure to maternal symptomatology (e.g., Monk et al., 2004). As with the earlier HLMs, each measure of maternal symptomatology was considered in a different model.

Based on prior work (DiPietro et al., 2004; Doyle et al., 2015), fetal sex, maternal age, gestational age (GA) at birth, pregnancy complications, and BMI (assessed at $30 \pm 2$ weeks gestation) were included as covariates in all fetal models.

We used linear regression models to examine the effect of prenatal medication on Bayley scores after controlling for maternal prenatal or postnatal mood. The following mood variables were included in separate models: average HRSD-17 and average HRSA during pregnancy and postpartum AUC of HRSD-17 and HRSA capturing symptoms occurring across the first year after delivery. As the majority of participants did not change their drug group over pregnancy, we considered medication group at the second testing session (30 weeks) in the primary analysis but also examined the effect of medication at the first and third testing sessions (24 and 36 weeks) in separate models. In all models, we adjusted for the same set of covariates used in the HLMs for fetal outcomes except maternal BMI. Finally, in an effort to determine if any medication or mood-linked difference in fetal development was associated with infant functioning in the postnatal period, we planned to test whether fetal development mediated the effect of medication on infant development using Sobel's test and the same covariates as already described.

Eighty participants $(50 \%$ of the $n=161)$ completed all three fetal sessions, $51(32 \%)$ completed two, and 30 (18\%) completed only one. Because HLM can accommodate missing outcome data, all participants were included in the analyses. Fetuses of women who completed the 24- and/or 30 -week fetal session versus those who also completed the 36-week fetal session had lower GA at birth $(p<.0001)$ and lower birth weight ( $p<.0009$, respectively). FM values more than 1.5 interquartile range below the first interquartile or above the third were excluded as outliers $(n=7,11$, and 11 from 24, 30, and 36 weeks, respectively). Improper 
administration of the BSID led to the exclusion of $n=5$ (6.25\%) infants for the MDI and $n=10(12.5 \%)$ for the PDI.

Finally, we reran all of our fetal and Bayley analyses with the following considerations: including only women who completed all three prenatal testing sessions $(n=80)$, controlling for birthweight corrected for GA instead of GA at birth, excluding participants who smoked $(n=20 ; 12.42 \%)$, took cardiovascular medication $(n=13 ; 8 \%)$, tested positive for a urine drug screen $(n=11$ individual women, 12 positive screens; $7 \%$ ), and took only non-SRI psychotropic medications $(n=20 ; 17 \%)$. We also examined results simultaneously removing all of these subjects from analyses.

All analyses were conducted using SAS 9.4 (SAS Institute Inc., Cary, NC) and the lavaan R-package (Rosseel, 2012). Continuous predictor variables were grand-mean centered prior to model parameterization. All hypotheses were tested at a 5\% significance level and for HLM models, Cohen's $d$ was computed (Bakeman, 2005) as a standardized effect size of the $t$ test, which quantifies the standardized mean difference between two medication groups relative to the pooled standard deviation.

\section{Results}

\section{Sample descriptives}

As shown in Table 1, the sample was generally socioeconomically advantaged and non-Hispanic White; $100 \%$ received prenatal care prior to 16 weeks gestation, and the overall preterm birth rate was $20.4 \%$. The four medication groups differed on birthweight with SRI Only and Non-SRI weighing the least $(p<.01)$.

Participants' depression and anxiety symptoms for each study session are shown in Table 2. As indicated, on average, the mood values did not change over pregnancy. With respect to clinically significant values on the HRSD-17 and HRSA (Ji et al., 2011), during pregnancy 30 (19\%) participants had HRSD-17 scores $>13$, and of those, 20 (66\%) were in one of the medication groups. For HRSA, 3 (2\%) women scored $>18$ and one was taking psychotropic medication.
As previously noted, within and across prenatal testing sessions, the two mood variables were highly correlated ( $r \mathrm{~s}=$ $.76-.82$, all $p<.0001$ ). The average prenatal mood scores were highly correlated with postnatal mood at 12 months $(r=.54-.58, p \mathrm{~s}<.0001)$. The drug screens were positive for amphetamine $(n=3)$, barbiturates $(n=4)$, cannabis $(n=4)$, and opiates $(n=2)$; these positive screens occurred in a total of 11 women (12 positive screens).

\section{Average fetal and infant development}

Fetuses on average showed the expected developmental trajectories such that FHR and FM decreased significantly across gestation and FHRV and coupling increased (see Table 3 and Figure 2). At 12 months, the mean infant BSID MDI score was in the low end of the normal range $(M=$ 89.92, $S D=11.16$, range $=55-115)$. The mean infant BSID PDI score was slightly below the normal range $(M=$ $84.1, S D=14.2$, range $=55-118)$. On the MDI, $n=23$ (30.67\% of the 75 valid administrations) scored at or below the level thought to indicate mild developmental delay (more than $1 S D$ below the mean; 84 or below) versus the expected $13.5 \%$ based on normative data for this measure; on the PDI, $n=29$ (41.43\% of the 70 valid administrations) fell below this criterion. Sixteen children scored at or below the level thought to indicate mild developmental delay on both the MDI and PDI. There were no differences in MDI or PDI results by term versus preterm birth status ( $p=.17$ and .46 , respectively).

\section{Psychotropic medication use and fetal development}

Based on HLM analyses of maternal medication use while controlling for depressive symptoms (measured using HRSD-17 scores averaged across 24-, 30-, and 36-week assessments) as well as the other covariates described in the Analysis section in relation to the level and trajectories of FHR, FHRV, FM, and coupling over gestation, there were significant findings only for FM: fetuses of women in the SRI Only group compared to No Medication had higher

Table 2. Maternal psychiatric symptoms during pregnancy and postpartum

\begin{tabular}{|c|c|c|c|c|}
\hline Symptom scale & $\begin{array}{c}24 \text { weeks gestation } \\
(n=137) \\
\text { Mean }(S D) \& \text { (range) }\end{array}$ & $\begin{array}{c}30 \text { weeks gestation } \\
(n=126) \\
\text { Mean }(S D) \& \text { (range) }\end{array}$ & $\begin{array}{c}36 \text { weeks gestation } \\
(n=106) \\
\text { Mean }(S D) \& \text { (range) }\end{array}$ & $\begin{array}{l}12 \text { months postpartum } \\
\quad(n=77) \\
\text { Mean }(S D) \& \text { (range) }\end{array}$ \\
\hline \multirow{3}{*}{ HRSD-17 } & $9.4(5.1)$ & $9.3(5.0)$ & $9.8(4.9)$ & \\
\hline & $(1-25)$ & $(1-25)$ & $(1-26)$ & \\
\hline & $7.4(4.3)$ & $8.4(4.6)$ & $8.7(4.6)$ & \\
\hline \multirow[t]{2}{*}{ HRSA } & $(0-24)$ & $(1-28)$ & $(1-26)$ & \\
\hline & & & $388.5(170.0)$ & 454.06 (206.7) \\
\hline \multirow[t]{2}{*}{ HRSD-17 AUC } & & & $(68.3-876.9)$ & $(77.4-1091.9)$ \\
\hline & & & $333.4(156.3)$ & $357.9(159.5)$ \\
\hline HRSA AUC & & & $(67.4-768.0)$ & $(71.7-776.5)$ \\
\hline
\end{tabular}

Note: HRSD-17, Hamilton Rating Scale for Depression (17 item). HRSA, Hamilton Rating Scale for Anxiety. AUC, area under the curve. 
Table 3. Means and standard deviations for fetal neurobehavioral development over gestation

\begin{tabular}{|c|c|c|c|c|c|c|c|c|}
\hline \multirow[b]{2}{*}{ Variable } & \multicolumn{2}{|c|}{$\begin{array}{l}\text { 1st testing session } \\
24 \text { weeks } \\
(n=139)\end{array}$} & \multicolumn{2}{|c|}{$\begin{array}{l}\text { 2nd testing session } \\
30 \text { weeks } \\
(n=125)\end{array}$} & \multicolumn{2}{|c|}{$\begin{array}{l}\text { 3rd testing session } \\
36 \text { weeks } \\
(n=108)\end{array}$} & \multicolumn{2}{|c|}{$\begin{array}{l}\text { Change } \\
\text { over time }\end{array}$} \\
\hline & Mean & $S D$ & Mean & $S D$ & Mean & $S D$ & Est & $p$ \\
\hline FHR & 146.10 & 7.30 & 142.59 & 8.03 & 141.11 & 8.87 & -2.45 & $<.0001$ \\
\hline FHRV & 6.69 & 2.72 & 7.20 & 3.00 & 8.10 & 4.54 & 0.72 & .0024 \\
\hline Coupling & 0.42 & 0.11 & 0.49 & 0.13 & 0.47 & 0.12 & 0.03 & .0009 \\
\hline Movement & 75991.64 & 58803.49 & 65067.61 & 55601.35 & 57619.40 & 57127.47 & -16317 & $<.0001$ \\
\hline
\end{tabular}

Note: The change over time estimates presented here are the unstandardized coefficient for the slope term from the unconditional hierarchical linear models used to examine change in fetal neurobehavioral development across testing sessions. FHR, fetal heart rate. FHRV, fetal heart rate variability.

FM at 24 and 30 weeks $(B=30821, p=.02$, Cohen's $d=$ $0.63 ; B=17667, p=.03$, Cohen's $d=0.36$, respectively). There were no significant results at 36 weeks $(p s>.20)$. With respect to pairwise comparisons, at 30 weeks, the SRI Only group had higher FM than the SRI+Non-SRI and Non-SRI groups ( $p=.03$ and .05, respectively; see Figure 3 ).
None of the covariates were significantly associated with FM. The variable that captured the time-varying effect of medication group was not associated with FM (all $p \mathrm{~s}>.4$ ). Very similar results were found when we controlled for maternal anxiety symptoms (measured using the HRSA scores averaged across 24-, 30-, and 36-week assessments) in place of
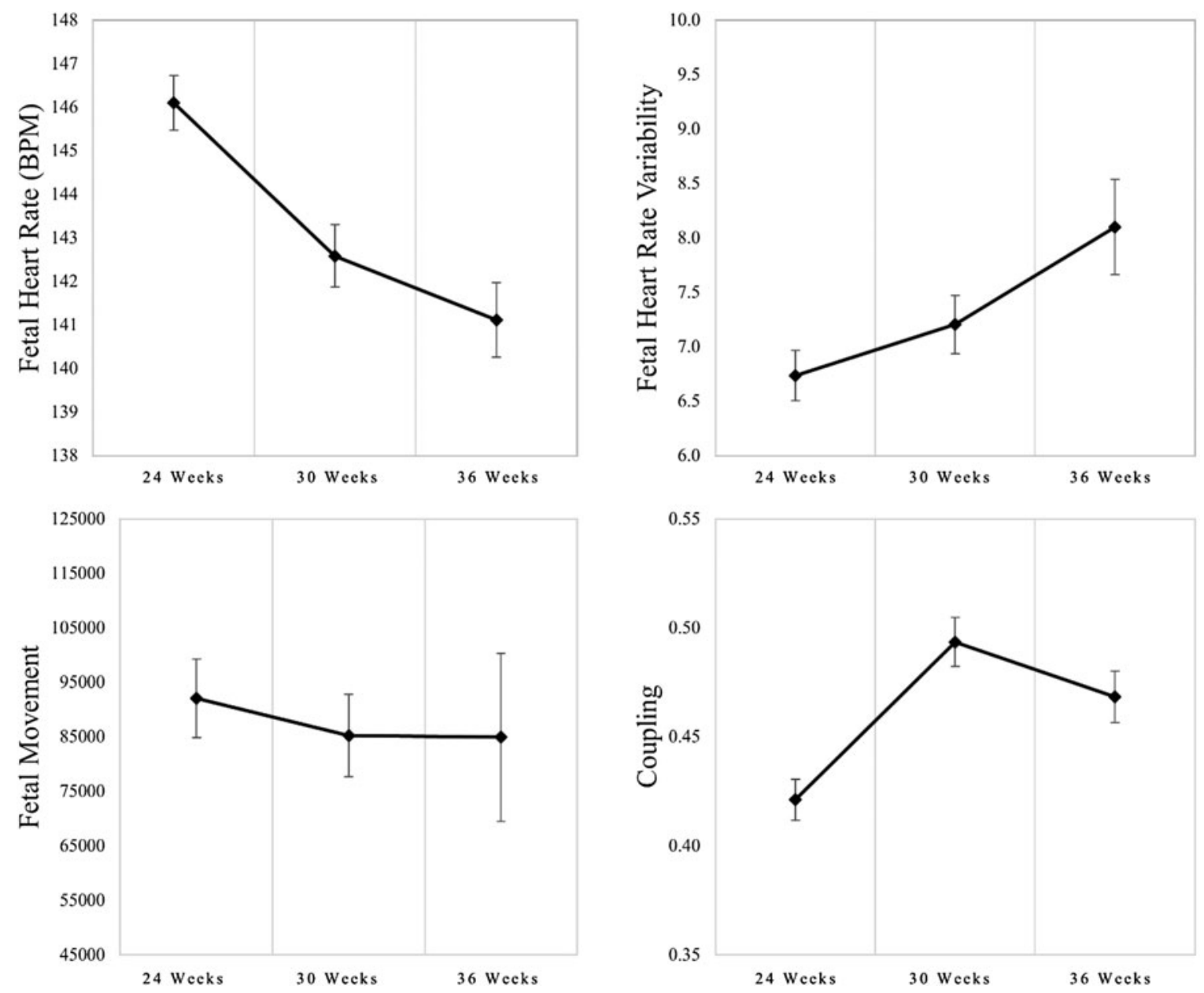

Figure 2. Four indices of fetal neurobehavioral development across gestation. Fetal heart rate; fetal heart rate variability (assessed as the standard deviation of fetal heart rate); fetal movement; and coupling (cross correlation of movement and heart rate changes). 


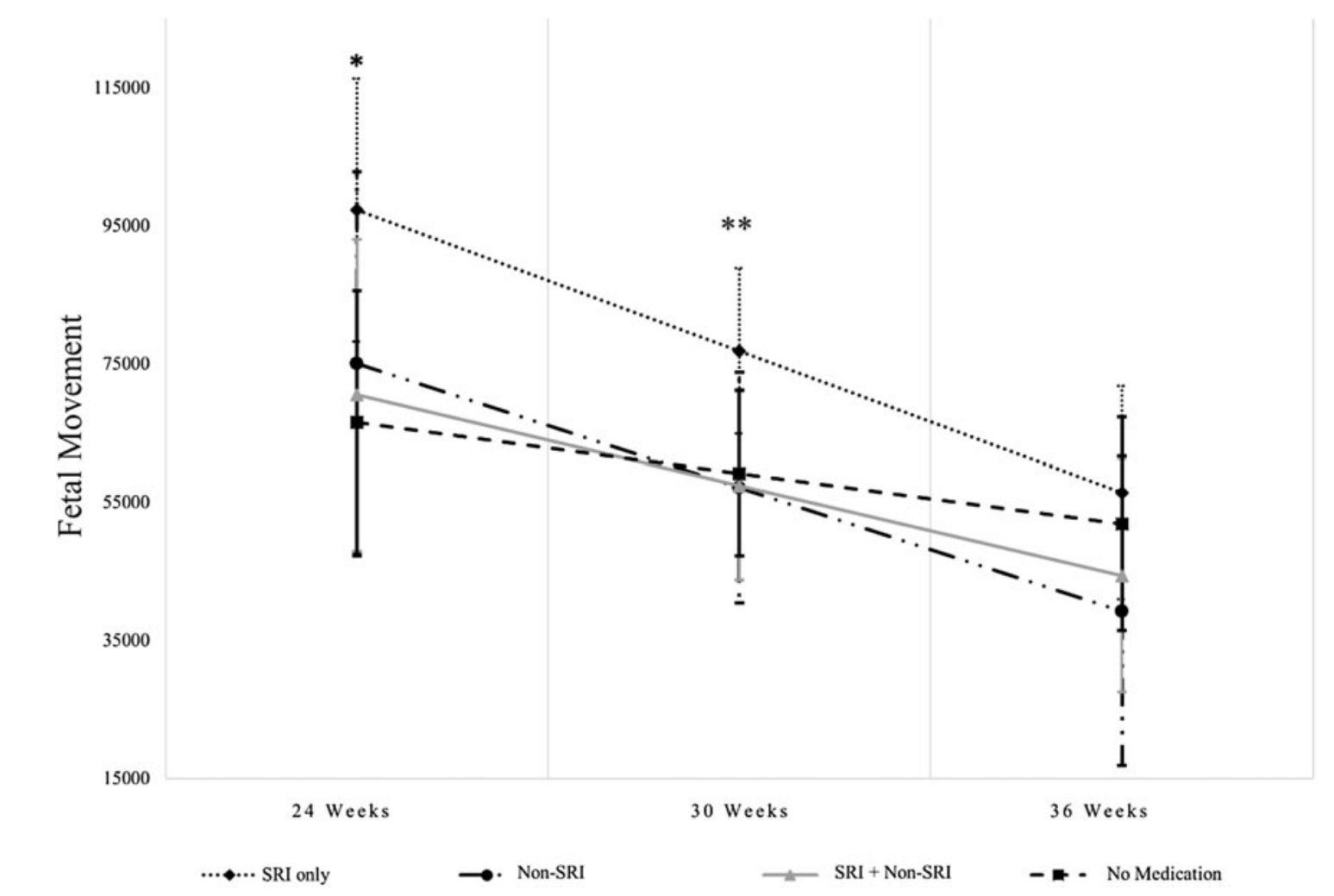

Figure 3. SRI-only use is associated with greater fetal movement. *At 24 weeks, the SRI Only group was significantly higher on fetal movement compared to the No Medication group ( $p=.02$ ).**At 30 weeks, the SRI Only group was significantly higher on fetal movement compared to the No Medication, SRI + Non-SRI, and Non-SRI groups ( $p=.03, .03$, and .05 , respectively).

depressive symptoms: fetuses of women in the SRI Only group compared to No Medication had higher FM at 24 and 30 weeks $(B=30756, p=.02$, Cohen's $d=0.63$; $B=17271, p=.03$, Cohen's $d=0.35$, respectively), but not at 36 weeks. At 30 weeks, the SRI Only group had higher FM than the SRI+Non-SRI and Non-SRI groups $(p \mathrm{~s}<.05)$.

\section{Psychotropic medication use and FHR reactivity}

At 24 and 30 weeks, there was, on average, no significant FHR change from baseline to the Stroop period. At 36 weeks, on average fetuses had a significant decrease in FHR from baseline to Stroop $(M=-1.97 \mathrm{bpm}, p<.05)$. There were no associations between maternal medication group and FHR change during maternal exposure to the Stroop task at any of the three testing sessions, regardless of the measure of maternal mood that was covaried (mean vs. AUC values of the HRSD-17 and HRSA), $p$ s ranged from .08 to .98 .

Women showed a significant increase in self-reported stress from the baseline to the Stroop periods ( $p s<.0001)$ at each testing session, confirming that the Stroop task was effective in inducing a stress response from the mother. There were no differences in changes in stress ratings from baseline to Stroop by medication group at any of the three testing sessions $(p s>.5)$.
Prenatal psychotropic medication use and infant development

Maternal medication use was not significantly associated with infant BSID PDI or MDI scores; this was true in models that covaried maternal depression or anxiety during the prenatal period (based on average HRSD-17 and HRSA scores) and during the postnatal period (based on HRSD-17 and HRSA AUC scores). Based on linear regression models of maternal medication group at each testing session in relation to infant PDI and MDI and controlling for prenatal mood (assessed using HRSD-17 and HRSA average values, considered in separate models), there were no medication effects ( $p s>.12$ ). Results of the regressions used to examine maternal medication group at 30 weeks gestation in relation to infant MDI and controlling for postpartum depression and anxiety using AUC calculations (with depression and anxiety AUC values considered in separate models) also suggest no significant effects of medication, $p \mathrm{~s}>.30$. SRI Only use assessed at 30 weeks versus No Medication was associated with lower PDI scores at trend level $(B \mathrm{~s}=-9.87,-9.75, p=.07$, .08 , Cohen's $d=-0.69,-0.68$, controlling for depression and anxiety, respectively). Postpartum mood was not a significant factor $(p s>.20)$. When we reran the analyses using medication group from the 24- and 36-week prenatal testing sessions, results remained at the trend level ( $p$ s ranging from .06 to 
.07). The following covariates were significant: pregnancy complications (yes/no) was positively associated with MDI outcome $(p s<.05)$; no covariates were associated with the PDI score $(p s>.05)$. Figure 4 presents mean MDI and PDI scores separately for each medication group.

\section{Additional analyses for fetal and infant models}

When we reran these analyses with additional considerations (including only women who completed all three prenatal testing sessions; $n=80$ ), controlling for birthweight corrected for GA at birth instead of GA at birth, excluding participants who smoked $(n=20 ; 12.42 \%)$, took cardiovascular medication $(n=13 ; 8 \%)$, tested positive for a urine drug screen $(n=11$ individual women, 12 positive screens; 7\%), and took only non-SRI psychotropic medications $(n=20 ; 17 \%)$, as well as simultaneously removing all of these subjects from analyses, our results were consistent with those reported above.

\section{Fetal development as a mediator of medication effects on infant development}

In an effort to test whether SRI-associated differences in FM are, in turn, associated with infant functioning in the postnatal period, we tested whether there was an indirect effect of SRI use on Bayley PDI scores, via greater FM. These analyses were based on data from individuals who contributed both prenatal data and infant Bayley data $(n=65)$. Using Sobel's test and the same covariates as in the Bayley models, there was no significant mediation effect associating SRI only use, FM, and the Bayley PDI scores (indirect effect $=-0.0003, p=.98$ ). However, we may have been underpowered to detect such an effect, so these results should be interpreted cautiously.

\section{Discussion}

Depression during pregnancy is relatively common, with an estimated $10 \%-15 \%$ prevalence rate (Gavin et al., 2005), yet the decision to use psychotropic medication remains complex given data indicating some associations with compromised child neurobehavioral development (Boukhris et al., 2016; Malm et al., 2016). However, for many of the studies of psychotropic medication effects, it is difficult to tease apart the relative contributions of the pharmacological treatment from maternal prenatal and postnatal psychopathology (Gur, Kim, \& Epperson, 2013; Oberlander \& Vigod, 2016). In this report, we studied pregnant women with a common psychiatric illness, MDD, actively engaged in clinical care with $70 \%$ taking a range of psychotropic medication, with SRI use as most common. We made serial assessments of neurobehavioral outcomes at the time of prenatal exposures, controlling for average symptoms of depression and anxiety, and at 12 months postpartum, controlling for prenatal and postpartum mood. Based on three evaluations across gestation that included five indices of fetal neurobehavioral development, medication use was, for the most part, not associated with fetal functioning. There were only findings with FM: SRI Only use versus No Medication was associated with more FM at 24 and 30 weeks gestation, though not at 36 weeks. In infancy, SRI Only use versus No Medication was associated with lower Bayley PDI at trend level and the decrement was approximately 10 points, which is less than $1 S D$ (15 points) on this standardized scale.

Maternal use of SRIs and greater FM has been reported previously (Mulder et al., 2011; Rurak et al., 2011; Salisbury, Ponder, Padbury, \& Lester, 2009). While Salisbury et al. (2009) found the increased FM at 26 and 36 gestational weeks, similar to our results, Mulder et al. (2011) showed this effect in early and middle pregnancy (15-19 and 27-29 gestational weeks) but not near term (37-39 weeks). In our results, it may be that fetuses not included in the 36-week testing session as a consequence of early birth were those who also had greater FM earlier in pregnancy. It is possible to speculate that SRI-associated increased FM is a prenatal manifestation of neonatal adaptation symptoms (transient respiratory distress, tremors, and irritability; Salisbury et al., 2016), though the significance of FM for long-term development is unclear (Mulder et al., 2011). In our results, FM was not associated with our trend result showing modestly reduced Bayley PDI following prenatal SRI exposure. We failed to support the hypothesized model of medication influences on infant behavior mediated by indices of fetal neurobehavioral development, though we may have been underpowered to detect such an effect. In one of the few other prospective studies of fetal to child development with $n=35$ healthy, nonmedicated mothers, greater FM was associated with less behavioral inhibition, itself a marker of risk for future psychopathology, in 24-month-olds (DiPietro et al., 2002).

Our trend finding associating SRI Only use with lower Bayley PDI score at 12 months is similar to those from other reports relating SRI use to lower scores on this Bayley development score at 6.5-16 months old (Casper et al., 2011; Hanley et al., 2013; Santucci et al., 2014). However, consistent with the Santucci et al. (2014) report, one with a large sample and rigorous control for maternal depression status and showing the lower scores were within the normal range, our SRI-associated reduction was less than $1 S D$ on the standardized Bayley scale. In the Santucci study, the PDI difference was no longer observed at 19.5 months.

With respect to polypharmacy and the impact of other psychotropic medications on fetal-infant development, none were detected. Our results are similar to Peng et al. (2013), who found that based on a case controlled study of $N=$ 152 infants prenatally exposed to atypical antipsychotics, any decrements in BSID cognitive and motor scores evident at 2 months dissipated by 12 . However, the smaller cell size, and combination of medications within those groups, may have precluded our ability to detect small effects.

On average, fetuses showed the expected developmental trajectories over gestation, a decrease in FHR and FM, and an increase in FHRV and coupling (DiPietro et al., 2004; Doyle et al., 2015). The values of these neurobehavioral in- 

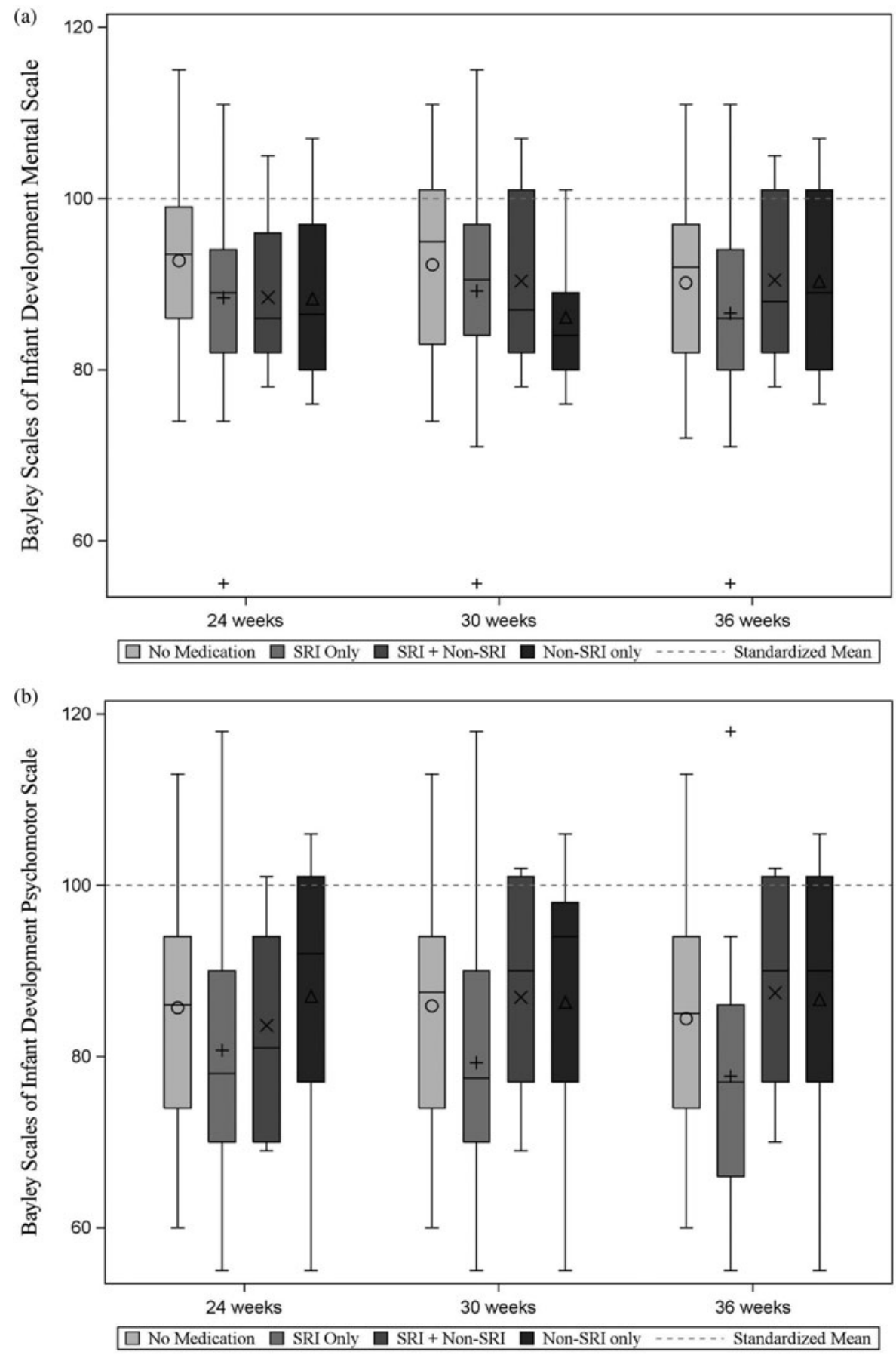

Figure 4. Average scores on the Bayley Scales of Infant Development (a) Mental Development Index and (b) Psychomotor Development Index, presented by medication group. The dashed line in each plot corresponds to the standardized mean $(M=100)$ for these scales.

dices were comparable to other reports (DiPietro et al., 2004; Doyle et al., 2015). In contrast, the sample's average Bayley scores at 12 months old were on the low end of the normal limits category and $\sim 30 \%-40 \%$ had scores indicating delayed performances for each of the two test domains, more than $2-3$ three times the rate that would be expected in the general population based on normative data for this measure.
Yet for both developmental time periods, prenatal to 1-year postnatal, adverse effects of maternal prenatal medication use on neurobehavioral development were largely undetected. Moreover, this is a sample of generally socioeconomically advantaged, non-Hispanic White women whose MDD was, for most, treated to euthymic levels, which are all characteristics that would predict typical development and Bayley 
scores. These unexpected findings suggest the following interpretation: mental health care directed primarily at women's psychiatric symptoms may not sufficiently address the additional unique needs of women parenting young infants (Goodman \& Garber, 2017). Psychosocial services that promote the mother-child relationship also may be needed to help optimize the postnatal environment and thereby positively affect child development (Forman et al., 2007).

Gur et al. (2013) have reasoned that since serotonin (5-HT) modulates neuroplasticity, the impact of SRI exposure on fetal brain development likely is significant, but may evolve over time and be context dependent. Similarly, Brummelte, Mc Glanaghy, Bonnin, and Oberlander, (2016) suggest that prenatal exposures that change 5-HT levels may act as plasticity rather than risk factor per se. Specifically, SRI exposure may alter the susceptibility to the environment, which, depending on the circumstances, could be deleterious or beneficial (Ellis \& Boyce, 2011; Pluess \& Belsky, 2011). With $55 \%$ of our sample taking SRIs at some point in pregnancy and $230 \%-40 \%$ of the infants having BSID scores in the mildly delayed category in the absence of significant SRI effects on fetal development, our findings suggest the possibility of enhanced receptivity to a somewhat compromised postnatal environment in some mother-infant dyads.

Strengths of this paper include a prospective study with multiple fetal assessments as well as infant assessment in a well-characterized, clinical sample of pregnant women with carefully documented psychiatric diagnoses, enabling us to ask the clinically relevant question of maternal use of a range of psychotropic medications in relation to fetal and child development. Our comparison of women who share a risk phenotype (a current primary diagnosis of MDD) but who differed in whether they were taking medication during pregnancy is an additional strength, as it represents a more ecologically valid comparison, one that more closely aligns with a depressed woman's risk assessment when considering medication use during pregnancy. An additional strength is extensive neurobehavioral testing during the time of prenatal exposure, and examination of both fetal and infant developmental outcomes.

This study also had limitations. Given the homogeneity of the sample's demographic characteristics, it is unknown if these results would generalize. Though focusing on women with a shared risk phenotype was important for our study design (both in terms of being able to create a more appropriate comparison group and for allowing our findings to be more directly compared to previously published reports focused on depression), it may also limit generalizability of our findings, given the frequently complex presentation of affective disorders, including high rates of comorbidity. Further, although individuals who are assigned the same SCID diagnosis are similar in many clinically meaningful ways, there remains substantial heterogeneity within DSM diagnostic categories, in terms of history, severity, course, and comorbidities, among other factors. In recognition of these issues, the current study incorporated measures of symptom severity assessed at multiple times across pregnancy, and considered both maternal depression and anxiety symptoms in all analyses. Nonetheless, adopting a transdiagnostic approach to examining the effects of medication and symptomology on fetal and infant development may yield additional information with clinical relevance. In the current analyses, we considered maternal depression and anxiety symptoms in separate models, motivated by concerns that putting them in the same model may result in the erroneous conclusion that one type of symptom was a larger contributor than the other (given their high correlation), when this study may not have been well suited to make such a distinction. This approach, however, makes it difficult to determine the extent to which the anxiety and depression effects presented here represent unique or overlapping findings. These results should be interpreted in light of this limitation.

The current study was somewhat unique in that it included data on both maternal medication use (including polypharmacy) and symptomatology, and examined whether these two in utero exposures exerted unique effects on fetal and child development. Though this approach likely represents an important first step in clarifying the nature of these effects, it does not properly capture the dynamic interplay between maternal symptomatology and medication use; a more comprehensive understanding of their effect requires examining how these two exposures may interact to predict child outcomes, or how different combinations of responses to medication (e.g., symptoms that remit while on medication vs. those that persist while on medication) may have different consequences for children. Unfortunately, the relatively euthymic nature of this largely medicated sample made it very difficult to explore these types of questions in the current study (as described above, only 20 women who were taking medication endorsed clinically significant symptomatology). Future research should pursue this line of thought.

The fetal monitoring sessions, though conducted by welltrained obstetrics nurses with extensive training, were relatively brief ( $5 \mathrm{~min}$ for each task period), and it is possible that we may not have detected small, yet clinically meaningful, differences in fetal neurobehavior during this time frame. The following points provided us with greater confidence that this time frame resulted in sufficient fetal data, though this still remains a concern: (a) we have successfully used this sampling window in previous research examining maternal symptomatology in relation to FHR reactivity (Monk et al., 2000, 2004); (b) incredibly brief assessments of fetal HR are used in a medical settings as a reliable index of fetal well-being; and (c) the current study was able to replicate expected developmental trajectories of all four fetal metrics, and report values that are comparable to published findings with different samples (DiPietro et al., 2004; Doyle et al., 2015). Though, of note, with the exception of FHR, there are not yet established normal parameters for the other fetal variables. Finally, as a single study with an outcome only to 12 months old (Gingrich et al., 2017; Johnson, Smith, Stowe, Newport, \& Brennan, 2016), these results must be replicated 
prior to formal inclusion in the risk/benefit assessment for psychotropic therapy during pregnancy.

Psychiatric illnesses are understood to be neurodevelopmental disorders (Insel \& Wang, 2010), with risk influences identified as early as the prenatal period (O'Connor et al., 2016), including that of maternal affective illness and the pharmacological treatment of it (O'Connor et al., 2016). In this study covering in utero and early postnatal development, associations between maternal prenatal psychotropic medication use and adverse offspring development were largely unsubstantiated. Rather, findings point to the potential importance of considering the well-being of the dyad, mother and child, in the context of maternal MDD, even when the woman's symptoms are well managed.

\section{References}

Ayad, M., \& Costantine, M. M. (2015). Epidemiology of medications use in pregnancy. Semininar in Perinatology, 39, 508-511. doi:10.1053/j.semperi.2015.08.002

Bakeman, R. (2005). Recommended effect size statistics for repeated measures designs. Behavior Research Methods, 37, 379-384.

Bank, A. M., Stowe, Z. N., Newport, D. J., Ritchie, J. C., \& Pennell, P. B. (2017). Placental passage of antiepileptic drugs at delivery and neonatal outcomes. Epilepsia, 58, e82-e86. doi:10.1111/epi.13733

Baser, I., Johnson, T. R., \& Paine, L. L. (1992). Coupling of fetal movement and fetal heart rate accelerations as an indicator of fetal health. Obstetrics and Gynecology, 80, 62-66.

Bobo, W. V., Davis, R. L., Toh, S., Li, D. K., Andrade, S. E., Cheetham, T. C., . . Cooper, W. O. (2012). Trends in the use of antiepileptic drugs among pregnant women in the US, 2001-2007: A medication exposure in pregnancy risk evaluation program study. Paediatric and Perinatal Epidemiology, 26, 578-588. doi:10.1111/ppe.12004

Boukhris, T., Sheehy, O., Mottron, L., \& Berard, A. (2016). Antidepressant use during pregnancy and the risk of autism spectrum disorder in children. JAMA Pediatrics, 170, 117-124. doi:10.1001/jamapediatrics. 2015.3356

Brummelte, S., McGlanaghy, E., Bonnin, A., \& Oberlander, T. F. (2016). Developmental changes in serotonin signaling: Implications for early brain function, behavior and adaptation. Neuroscience. Advance online publication. doi:10.1016/j.neuroscience.2016.02.037

Casper, R. C., Gilles, A. A., Fleisher, B. E., Baran, J., Enns, G., \& Lazzeroni, L. C. (2011). Length of prenatal exposure to selective serotonin reuptake inhibitor (SSRI) antidepressants: Effects on neonatal adaptation and psychomotor development. Psychopharmacology, 217, 211-219. doi: $10.1007 / \mathrm{s} 00213-011-2270-\mathrm{Z}$

Clements, C. C., Castro, V. M., Blumenthal, S. R., Rosenfield, H. R., Murphy, S. N., Fava, M., . . . Perlis, R. H. (2015). Prenatal antidepressant exposure is associated with risk for attention-deficit hyperactivity disorder but not autism spectrum disorder in a large health system. Molecular Psychiatry, 20, 727-734. doi:10.1038/mp.2014.90

Cummings, C., Stewart, M., Stevenson, M., Morrow, J., \& Nelson, J. (2011). Neurodevelopment of children exposed in utero to lamotrigine, sodium valproate and carbamazepine. Archives of Disease in Childhood, 96, 643-647. doi:10.1136/adc.2009.176990

Dalton, K. J., Dawes, G. S., \& Patrick, J. E. (1983). The autonomic nervous system and fetal heart rate variability. American Journal of Obstetrics and Gynaecology, 146, 456-462.

DiPietro, J. A., Bornstein, M. H., Costigan, K. A., Pressman, E. K., Hahn, C. S., Painter, K., . . Y Yi, L. J. (2002). What does fetal movement predict about behavior during the first two years of life? Developmental Psychobiology, 40, 358-371. doi:10.1002/dev.10025

DiPietro, J. A., Bornstein, M. H., Hahn, C. S., Costigan, K., \& Achy-Brou, A. (2007). Fetal heart rate and variability: Stability and prediction to developmental outcomes in early childhood. Child Development, 78, 1788-1798. doi:10.1111/j.1467-8624.2007.01099.x

DiPietro, J. A., Caulfield, L., Costigan, K. A., Merialdi, M., Nguyen, R. H., Zavaleta, N., \& Gurewitsch, E. D. (2004). Fetal neurobehavioral development: A tale of two cities. Developmental Psychology, 40, 445-456. doi:10.1037/0012-1649.40.3.445

DiPietro, J. A., Hodgson, D. M., Costigan, K. A., \& Johnson, T. R. (1996). Fetal antecedents of infant temperament. Child Development, 67, 25682583.

Dipietro, J. A., Irizarry, R. A., Hawkins, M., Costigan, K. A., \& Pressman, E. K. (2001). Cross-correlation of fetal cardiac and somatic activity as an indicator of antenatal neural development. American Journal of Obstetrics and Gynecology, 185, 1421-1428. doi:10.1067/mob.2001.119108

DiPietro, J. A., Kivlighan, K. T., Costigan, K. A., Rubin, S. E., Shiffler, D. E., Henderson, J. L., \& Pillion, J. P. (2010). Prenatal antecedents of newborn neurological maturation. Child Development, 81, 115-130. doi:10.1111/ j.1467-8624.2009.01384.x

Doussard-Roosevelt, J. A., McClenny, B. D., \& Porges, S. W. (2001). Neonatal cardiac vagal tone and school-age developmental outcome in very low birth weight infants. Developmental Psychobiology, 38, 56-66.

Doyle, C., Werner, E., Feng, T., Lee, S., Altemus, M., Isler, J. R., \& Monk, C. (2015). Pregnancy distress gets under fetal skin: Maternal ambulatory assessment and sex differences in prenatal development. Developmental Psychobiology, 57, 607-625. doi:10.1002/dev.21317

Ellis, B. J., \& Boyce, W. T. (2011). Differential susceptibility to the environment: Toward an understanding of sensitivity to developmental experiences and context. Development and Psychopathology, 23, 1-5. doi:10.1017/s095457941000060x

First, M. B., Spitzer, R. L., Gibbon, M., \& Williams, J. B. W. (1997). Structured Clinical Interview for DSM-IV axis I disorders-Non-patient edition. New York: New York State Psychiatric Institute, Biometrics Research Department.

Forman, D. R., O’Hara, M. W., Stuart, S., Gorman, L. L., Larsen, K. E., \& Coy, K. C. (2007). Effective treatment for postpartum depression is not sufficient to improve the developing mother-child relationship. Development and Psychopathology, 19, 585-602. doi:10.1017/S0954579407070289

Freeman, R. K., Garite, T. J., Nageotte, M. P., \& Miller, L. A. (2012). Fetal heart rate monitoring (4th ed.). Philadelphia: Lippincott Williams \& Wilkins.

Gavin, N. I., Gaynes, B. N., Lohr, K. N., Meltzer-Brody, S., Gartlehner, G., \& Swinson, T. (2005). Perinatal depression: A systematic review of prevalence and incidence. Obstetrics and Gynecology, 106, 1071-1083.

Gingrich, J. A., Malm, H., Ansorge, M. S., Brown, A., Sourander, A., Suri, D., ... Weissman, M. M. (2017). New insights into how serotonin selective reuptake inhibitors shape the developing brain. Birth Defects Research, 109, 924-932. doi:10.1002/bdr2.1085

Goodman, S. H., \& Garber, J. (2017). Evidence-based interventions for depressed mothers and their young children. Child Development, 88, 368-377. doi:10.1111/cdev.12732

Grote, N. K., Bridge, J. A., Gavin, A. R., Melville, J. L., Iyengar, S., \& Katon, W. J. (2010). A meta-analysis of depression during pregnancy and the risk of preterm birth, low birth weight, and intrauterine growth restriction. Archives of General Psychiatry, 67, 1012-1024. doi:10.1001/archgenpsychiatry.2010.111

Gur, T. L., Kim, D. R., \& Epperson, C. N. (2013). Central nervous system effects of prenatal selective serotonin reuptake inhibitors: Sensing the signal through the noise. Psychopharmacology, 227, 567-582. doi:10.1007/ s00213-013-3115-8

Hamilton, M. (1959). The assessment of anxiety states by rating. British Journal of Medical Psychology, 32, 50-55.

Hamilton, M. (1960). A rating scale for depression. Journal of Neurology, Neurosurgery, \& Psychiatry, 23, 56-62.

Hanley, G. E., Brain, U., \& Oberlander, T. F. (2013). Infant developmental outcomes following prenatal exposure to antidepressants, and maternal depressed mood and positive affect. Early Human Development, 89, 519-524. doi:10.1016/j.earlhumdev.2012.12.012

Insel, T. R., \& Wang, P. S. (2010). Rethinking mental illness. Journal of the American Medical Association, 303, 1970-1971. doi:10.1001/jama. 2010.555

Ji, S., Long, Q., Newport, D. J., Na, H., Knight, B., Zach, E. B., . . Stowe, Z. N. (2011). Validity of depression rating scales during pregnancy and the postpartum period: Impact of trimester and parity. Journal of Psychiatric Research, 45, 213-219. doi:10.1016/j.jpsychires.2010.05.017 
Johnson, K. C., LaPrairie, J. L., Brennan, P. A., Stowe, Z. N., \& Newport, D. J. (2012). Prenatal antipsychotic exposure and neuromotor performance during infancy. Archives of General Psychiatry, 69, 787-794. doi:10. 1001/archgenpsychiatry.2012.160

Johnson, K. C., Smith, A. K., Stowe, Z. N., Newport, D. J., \& Brennan, P. A. (2016). Preschool outcomes following prenatal serotonin reuptake inhibitor exposure: Differences in language and behavior, but not cognitive function. Journal of Clinical Psychiatry, 77, e176-e182. doi:10.4088/JCP.14m09348

Lusby, C. M., Goodman, S. H., Bell, M. A., \& Newport, D. J. (2014). Electroencephalogram patterns in infants of depressed mothers. Developmental Psychobiology, 56, 459-473. doi:10.1002/dev.21112

Malm, H., Brown, A. S., Gissler, M., Gyllenberg, D., Hinkka-Yli-Salomaki, S., McKeague, I. W., . . . Sourander, A. (2016). Gestational exposure to selective serotonin reuptake inhibitors and offspring psychiatric disorders: A national register-based study. Journal of the American Academy of Child \& Adolescent Psychiatry, 55, 359-366. doi:10.1016/j.jaac.2016.02.013

Monk, C., Feng, T., Lee, S., Krupska, I., Champagne, F. A., \& Tycko, B. (2016). Distress during pregnancy: Epigenetic regulation of placenta glucocorticoid-related genes and fetal neurobehavior. American Journal of Psychiatry, 173, 705-713. doi:10.1176/appi.ajp.2015.15091171

Monk, C., Fifer, W. P., Myers, M. M., Bagiella, E., Duong, J. K., Chen, I. S., ... Arman, A. (2011). Effects of maternal breathing rate, psychiatric status, and cortisol on fetal heart rate. Developmental Psychobiology, 53, 221-233. doi:10.1002/dev.20513

Monk, C., Fifer, W. P., Myers, M. M., Sloan, R. P., Trien, L., \& Hurtado, A. (2000). Maternal stress responses and anxiety during pregnancy: Effects on fetal heart rate. Developmental Psychobiology, 36, 67-77. doi:10.1002/(SICI)1098-2302(200001)36:1<67::AID-DEV7>3.0. $\mathrm{CO} ; 2-\mathrm{C}$

Monk, C., Myers, M. M., Sloan, R. P., Werner, L., Jeon, J., Tager, F., \& Fifer, W. P. (2004). Fetal heart rate reactivity differs by women's psychiatric status: An early marker for developmental risk? Journal of the American Academy of Child \& Adolescent Psychiatry, 43, 283-290.

Monk, C., Newport, D. J., Korotkin, J. H., Long, Q., Knight, B., \& Stowe, Z. N. (2012). Uterine blood flow in a psychiatric population: Impact of maternal depression, anxiety, and psychotropic medication. Biological Psychiatry, 72, 483-490. doi:10.1016/j.biopsych.2012.05.006

Mulder, E. J., Ververs, F. F., de Heus, R., \& Visser, G. H. (2011). Selective serotonin reuptake inhibitors affect neurobehavioral development in the human fetus. Neuropsychopharmacology, 36, 1961-1971. doi:10.1038/ npp.2011.67

Newport, D. J., Calamaras, M. R., DeVane, C. L., Donovan, J., Beach, A. J., Winn, S., . . . Stowe, Z. N. (2007). Atypical antipsychotic administration during late pregnancy: Placental passage and obstetrical outcomes. American Journal of Psychiatry, 164, 1214-1220. doi:10.1176/appi.ajp.2007. 06111886

Oberlander, T. F., \& Vigod, S. N. (2016). Developmental effects of prenatal selective serotonin reuptake inhibitor exposure in perspective: Are we comparing apples to apples? Journal of the American Academy of Child \& Adolescent Psychiatry, 55, 351-352. doi:10.1016/j.jaac.2016.02.012

O’Connor, T. G., Monk, C., \& Burke, A. S. (2016). Maternal affective illness in the perinatal period and child development: Findings on developmental timing, mechanisms, and intervention. Current Psychiatry Reports, 18, 24. doi:10.1007/s11920-016-0660-y
Peng, M., Gao, K., Ding, Y., Ou, J., Calabrese, J. R., Wu, R., \& Zhao, J. (2013). Effects of prenatal exposure to atypical antipsychotics on postnatal development and growth of infants: A case-controlled, prospective study. Psychopharmacology, 228, 577-584. doi:10.1007/s00213-0133060-6

Pluess, M., \& Belsky, J. (2011). Prenatal programming of postnatal plasticity? Development and Psychopathology, 23, 29-38. doi:10.1017/ S0954579410000623

Posner, J., Cha, J., Roy, A. K., Peterson, B. S., Bansal, R., Gustafsson, H. C., .. . Monk, C. (2016). Alterations in amygdala-prefrontal circuits in infants exposed to prenatal maternal depression. Translational Psychiatry, 6, e935. doi:10.1038/tp.2016.146

Putnam, K. T., Wilcox, M., Robertson-Blackmore, E., Sharkey, K., Bergink, V., Munk-Olsen, T., . . . Treatment, C. (2017). Clinical phenotypes of perinatal depression and time of symptom onset: Analysis of data from an international consortium. Lancet Psychiatry, 4, 477-485. doi:10. 1016/S2215-0366(17)30136-0

Rosseel, Y. (2012). lavaan: An R package for structural equation modeling. Journal of Statistical Software, 48, 1-36.

Rurak, D., Lim, K., Sanders, A., Brain, U., Riggs, W., \& Oberlander, T. F. (2011). Third trimester fetal heart rate and Doppler middle cerebral artery blood flow velocity characteristics during prenatal selective serotonin reuptake inhibitor exposure. Pediatric Research, 70, 96-101. doi:10.1038/ pr.2011.321

Salisbury, A. L., O'Grady, K. E., Battle, C. L., Wisner, K. L., Anderson, G. M., Stroud, L. R., . . Lester, B. M. (2016). The roles of maternal depression, serotonin reuptake inhibitor treatment, and concomitant benzodiazepine use on infant neurobehavioral functioning over the first postnatal month. American Journal of Psychiatry, 173, 147-157. doi:10.1176/appi.ajp.2015.14080989

Salisbury, A. L., Ponder, K. L., Padbury, J. F., \& Lester, B. M. (2009). Fetal effects of psychoactive drugs. Clinics in Perinatology, 36, 595-619. doi:10.1016/j.clp.2009.06.002

Santucci, A. K., Singer, L. T., Wisniewski, S. R., Luther, J. F., Eng, H. F., Dills, J. L., . . . Wisner, K. L. (2014). Impact of prenatal exposure to serotonin reuptake inhibitors or maternal major depressive disorder on infant developmental outcomes. Journal of Clinical Psychiatry, 75, 1088 1095. doi:10.4088/JCP.13m08902

Santucci, A. K., Singer, L. T., Wisniewski, S. R., Luther, J. F., Eng, H. F., Sit, D. K., \& Wisner, K. L. (2017). One-year developmental outcomes for infants of mothers with bipolar disorder. Journal of Clinical Psychiatry. Advance online publication. doi:10.4088/JCP.15m 10535

Sujan, A. C., Rickert, M. E., Oberg, A. S., Quinn, P. D., Hernandez-Diaz, S., Almqvist, C., . . . D’Onofrio, B. M. (2017). Associations of maternal antidepressant use during the first trimester of pregnancy with preterm birth, small for gestational age, autism spectrum disorder, and attention-deficit/hyperactivity disorder in offspring. Journal of the American Medical Association, 317, 1553-1562. doi:10.1001/ jama.2017.3413

Werner, A., Myers, M. M., Fifer, W. P., Cheng, B., Fang, Y., Allen, R., \& Monk, C. (2007). Prenatal predictors of infant temperament. Developmental Psychobiology, 49, 474-484.

Williams, J. B. (1988). A structured interview guide for the Hamilton Depression Rating Scale. Archives of General Psychiatry, 45, 742-747. 